\title{
Dynamic Uplink Power Control for Cellular Radio Systems over Fast Fading Channel
}

\author{
Liang Dong and Guanghan Xu \\ Department of Electrical and Computer Engineering \\ The University of Texas at Austin \\ Austin, TX 78712, USA \\ \{dong, xu\}@ece.utexas.edu
}

\begin{abstract}
In cellular wireless CDMA systems, it is required that the base station receives constant power signals from mobile users, in order to guarantee link quality. This is achieved by properly adjusting the uplink transmitted power of each mobile. Usually, the base station is responsible for informing each individual mobile of the power adjustment. However, when the radio channel varies drastically under fast fading, the power control signaling from the base station may not be appropriate for the current uplink. In the proposed power control scheme, the mobile transmits at variable power levels based on the uplink channel prediction. The prediction accuracy is improved by exploiting the relationship between the access channel and the traffic channel. Simulation results show that this scheme combats the fast fading channel effect, hence increasing the system performance.
\end{abstract}

\section{Introduction}

In cellular wireless CDMA communication systems, mobile users adapt to time varying radio channels by regulating transmitted powers. The power control on the uplink attempts to adjust the transmitted power of each mobile such that the base station (BS) receives signals from each mobile with equal and constant nominal received power [8]. Optimal power control algorithms aim at minimizing the power transmitted by each mobile while maintaining required link quality.

The power control algorithms that have been developed to date assume slow fading radio channels. The channel characteristics vary slowly over several uplink and downlink frame periods. In TDD systems, the BS sends power control signaling to an individual mobile according to the received signal power it measures from previous uplinks. However, when the mobile moves in the propagation envi- ronment, such that Doppler spread and multipath scattering cause rapid fluctuation in the received signal, the radio channel undergoes Rayleigh fading and varies drastically within several frame periods [3]. This poses significant error in traditional power control, in which the mobile adjusts power based on the channel characteristics of the previous uplinks. Under fast fading, the control signaling from the BS is not suitable for the current uplink. Also, the constant transmitted power will not be able to cope with the deep fades within an uplink frame period.

In the proposed power control scheme, the mobile transmits at variable power levels based on the uplink traffic channel prediction. This control is open loop in the sense that the mobile user does not need BS signaling for power adjustment. Operating in TDD mode, the mobile extracts the most recent downlink channel from the received signal, and predicts the channel for uplink transmission. The AR model is employed for channel prediction. The mobile dynamically adjusts the transmitted power within an uplink frame according to the channel variation, such that the fast fading effect is compensated.

When an antenna array is used at the BS, downlink beamforming technique exploits the spatial diversity of the mobiles to increase system capacity [4]. However, imperfect beamforming increases prediction error. In this work, the uplink channel prediction relies on both the omnidirectional access channel (ACH) and the user-specified traffic channel (TCH) to improve accuracy.

\section{Channel and Signal Model}

\subsection{Propagation Environment}

A BS with an antenna array, serving multiple mobile users emitting narrowband signals is considered as in Fig. 1. The multipaths which cause random phase change and angle spread at the receiver are a result of the following two 


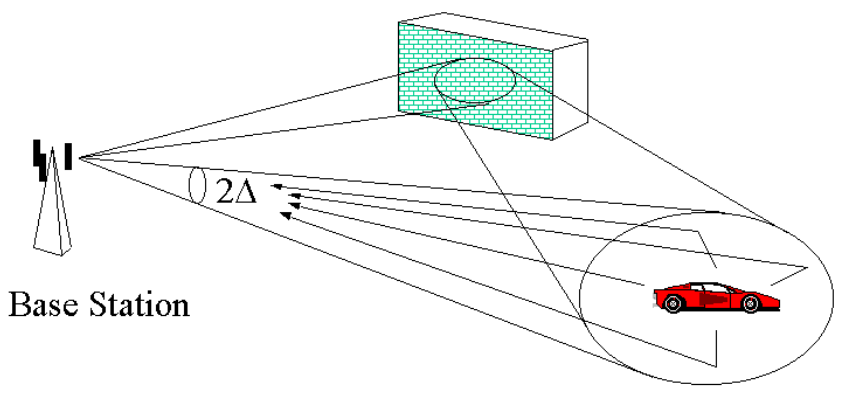

Mobile User

\section{Figure 1. Multipaths caused by local and re- mote scatterers}

mechanisms. First, the transmitted signals are scattered by objects local to the transmitter. Secondly, the scattering ray bundles are reflected or diffracted by objects remote to the transmitter. For local-scattering paths, it is reasonable to assume that the signal energy is dominant within a small spreading angle $\Delta$ with a uniform distribution. The reflection or diffraction by remote objects cause significant multipath delays and large angle spread.

Due to the motion of the mobile or the scatterers, the BS received signals experience Doppler frequency spread. The increased mobility results in fast fading in which the channel exhibits rapid temporal variations, such that the performance of the cellular radio system is substantially degraded. Fast-fading channels encountered in practice exhibit Doppler spreads on the order of 100-200 Hz [5]. With carrier frequency $2 \mathrm{GHz}$ and Doppler spread $100 \mathrm{~Hz}$, the range of the channel envelope within $5 \mathrm{~ms}$ frame of uplink and downlink can be as large as $10 \mathrm{~dB}$.

\subsection{Vector Channel Model}

When an M-element antenna array is mounted on the BS, the channel between the BS and the mobile is modeled using the Vector Channel Impulse Response (VCIR). Without considering local scattering effect,

$$
\mathbf{h}(t, \tau)=\sum_{i=0}^{L-1} \alpha_{i}(t, \tau) \mathbf{a}\left(\theta_{i}\right) \delta\left(t-\tau_{i}(\tau)\right)
$$

where $\alpha_{i}, \tau_{i}, \mathbf{a}\left(\theta_{i}\right)$ are the complex amplitude, path delay and steering vector associated with DOA $\left(\theta_{i}\right)$ of the $i^{t h}$ multipath component. There are a total of $\mathrm{L}$ multipath components. The complex amplitude can be expressed as

$$
\alpha_{i}(t, \tau)=\rho_{i}(\tau) e^{j\left(2 \pi f_{i}(\tau) t+\Psi_{i}(\tau)\right)}
$$

where $\rho_{i}, f_{i}$, and $\Psi_{i}$ are the path gain, Doppler shift and phase offset. Assume that the parameters $\rho_{i}, f_{i}, \Psi_{i}$ and $\tau_{i}$ remain constant during the short period of several TDD frames under consideration. Thus, the BS received baseband data vector from user $j$ is given by

$$
\begin{aligned}
\mathbf{x}(t) & =\mathbf{h}(t, \tau) * u(t)+\mathbf{n}(t) \\
& =\sum_{i=0}^{L-1} \alpha_{i}(t) \mathbf{a}\left(\theta_{i}\right) u\left(t-\tau_{i}\right)+\mathbf{n}(t) \\
& =u\left(t-\tau_{0}\right) \mathbf{A}^{(j)}(t)+\mathbf{n}(t)
\end{aligned}
$$

where, $u(t)$ is the baseband transmitted signal which depends on the information-bearing symbol and the modulation waveform, $\mathbf{n}(t)$ is zero-mean, complex AWGN vector. The last step of (3) makes the narrowband channel assumption that $\tau_{i} \approx \tau_{0} . \mathbf{A}^{(j)}(t)=\sum_{i=0}^{L-1} \alpha_{i}(t) \mathbf{a}\left(\theta_{i}\right)$.

Consider locally scattered paths from one nominal DOA $\theta$, the received data vector becomes [1]

$$
\begin{aligned}
\mathbf{x}(t) & =\sum_{k=0}^{N-1} \alpha_{k}(t) \mathbf{a}\left(\theta+\tilde{\theta}_{k}\right) u\left(t-\tau_{k}\right)+\mathbf{n}(t) \\
& =u\left(t-\tau_{0}\right) \mathbf{A}_{i}^{(j)}(t)+\mathbf{n}(t)
\end{aligned}
$$

where $\mathrm{N}$ is the total number of scattered paths within small angle spread $\Delta$, and $\theta+\tilde{\theta}_{k}$ is the arrival angle of the $k^{t h}$ scattered path. The delay spread of scattered paths are usually insignificant because of the small scattering radius. With $\left|\tilde{\theta}_{k}\right| \leq \Delta$, a first-order Taylor series expansion yields

$$
\begin{aligned}
\mathbf{A}_{i}^{(j)} & =\sum_{k=0}^{N-1} \alpha_{k} \mathbf{a}\left(\theta+\tilde{\theta}_{k}\right) \\
& \cong \sum_{k=0}^{N-1} \alpha_{k}\left[\mathbf{a}(\theta)+\tilde{\theta}_{k} \mathbf{d}(\theta)\right] \\
& =\left(\sum_{k=0}^{N-1} \alpha_{k}\right) \mathbf{a}(\theta)+\left(\sum_{k=0}^{N-1} \alpha_{k} \tilde{\theta}_{k}\right) \mathbf{d}(\theta)
\end{aligned}
$$

where, $\mathbf{d}(\theta)=\partial \mathbf{a}(\theta) / \partial \theta$ is the gradient.

Combining the effect of local scattering with that of large-angle reflection in (3) gives

$$
\begin{aligned}
\mathbf{x}(t) & =\sum_{i=0}^{L-1}\left[\gamma_{i}(t) \mathbf{a}\left(\theta_{i}\right)+\psi_{i}(t) \mathbf{d}\left(\theta_{i}\right)\right] u\left(t-\tau_{i}\right)+\mathbf{n}(t) \\
& =\mathbf{b}_{j}(t) u(t)+\mathbf{n}(t)
\end{aligned}
$$

where

$$
\begin{gathered}
\mathbf{b}_{j}(t)=\sum_{i=0}^{L-1}\left[\gamma_{i}(t) \mathbf{a}\left(\theta_{i}\right)+\psi_{i}(t) \mathbf{d}\left(\theta_{i}\right)\right] \\
\gamma_{i}(t)=\sum_{k=0}^{N_{i}-1} \alpha_{i k}(t), \quad \psi_{i}(t)=\sum_{k=0}^{N_{i}-1} \alpha_{i k}(t) \tilde{\theta}_{i k}
\end{gathered}
$$

$\mathbf{b}_{j}(t)$, the composite multiplicative channel, is called the spatial signature of the narrowband channel for user $\mathrm{j}$. 


\subsection{Mobile Received Signal}

In TDD systems, radio channels for uplink and downlink are reciprocal. The signal transmitted by the BS antenna array can be described as

$$
\mathbf{u}(t)=\sum_{d=0}^{D} \mathbf{w}_{d} u_{d}(t)
$$

where, $\mathbf{w}_{d}$ 's are the beam-forming weight vectors. $\mathbf{w}_{0}=$ $\mathbf{1}(=\operatorname{ones}(M, 1))$ is for the omni-directional access channel $(\mathrm{ACH})$, whereas $\mathbf{w}_{d}, d \in\{1, \ldots, D\}$, is for the traffic channel (TCH) of each user. D is the number of mobile users. $u_{0}(t)$ is the common access signal, and $u_{d}(t)$ is the signal for user $d$. Therefore the signal received by user $j$ is

$$
x_{j}(t)=\mathbf{b}_{j}^{T}(t) \mathbf{u}(t)+n_{j}(t)
$$

Suppose the signal-to-interference-and-noise-ratio (SINR) for user $j$ is above the satisfactory threshold, then the received signal can be further expressed as

$$
\begin{aligned}
x_{j}(t)= & \mathbf{b}_{j}^{T}(t) \mathbf{w}_{j} u_{j}(t)+\mathbf{b}_{j}^{T}(t) \mathbf{w}_{0} u_{0}(t) \\
& +\sum_{\substack{d \neq 0, d \neq j \\
=}} \mathbf{b}_{j}^{T}(t) \mathbf{w}_{d} u_{d}(t)+n_{j}(t) \\
= & \mathbf{b}_{j}^{T}(t) \mathbf{w}_{j} u_{j}(t)+\mathbf{b}_{j}^{T}(t) \mathbf{1} u_{0}(t)+\tilde{n}_{j}(t)(10)
\end{aligned}
$$

Interference from signals for other users is absorbed into the noise component $\tilde{n}_{j}$. The two signal parts in (10) can be separated by CDMA orthogonal codes. Therefore, after detecting $u_{j}(t)$ and $u_{0}(t)$, the mobile user can extract the $\mathrm{TCH}$ as $\mathbf{b}_{j}^{T}(t) \mathbf{w}_{j}$ and the ACH as $\mathbf{b}_{j}^{T}(t) \mathbf{1}$. Now the task is to predict the $\mathrm{TCH}$ in the following uplink frame for the purpose of dynamic power control.

\section{Dynamic Uplink Power Control Based on Channel Prediction}

\subsection{ACH Assisted Channel Prediction}

The traffic channel $c(t)$ of user $j$ is defined as

$$
\begin{aligned}
c(t) & =\mathbf{w}_{j}^{T} \mathbf{b}_{j}(t) \\
& =\mathbf{w}_{j}^{T} \sum_{i=0}^{L-1}\left[\gamma_{i}(t) \mathbf{a}\left(\theta_{i}\right)+\psi_{i}(t) \mathbf{d}\left(\theta_{i}\right)\right] \\
& =\mathbf{w}_{j}^{T} \sum_{i=0}^{L-1} \gamma_{i}(t)\left[\mathbf{a}\left(\theta_{i}\right)+\tilde{\mathbf{d}}_{i}\right] \\
& =\mathbf{w}_{j}^{T}[\mathbf{A}+\mathbf{D}] \Gamma
\end{aligned}
$$

where,

$$
\mathbf{A}=\left[\mathbf{a}\left(\theta_{0}\right), \ldots, \mathbf{a}\left(\theta_{L-1}\right)\right], \quad \mathbf{D}=\left[\tilde{\mathbf{d}}_{0}, \ldots, \tilde{\mathbf{d}}_{L-1}\right],
$$

$$
\Gamma=\left[\gamma_{0}(t), \ldots, \gamma_{L-1}(t)\right]^{T}
$$

and

$$
\tilde{\mathbf{d}}_{i}=\frac{\psi_{i}(t)}{\gamma_{i}(t)} \mathbf{d}\left(\theta_{i}\right)
$$

Note that $\mathbf{w}_{j}$ and $\mathbf{A}$ are constant vector and matrix, $\|\mathbf{D}\|$ is of order $O(\Delta)$ and it is assumed that the derivatives exist and that they are bounded. From (2) and (7) we see that $\gamma_{i}$ 's are of harmonic forms that consist of a superposition of complex exponentials. As a result, sharp peaks are the predominant feature of the power spectrum of channel $c(t)$. Therefore, the autoregressive (AR) model is most appropriate for the channel [2]. The sharp peaks correspond to the poles near the unit circle. The AR model is closely tied to linear prediction, and the prediction coefficients $\mathbf{a}_{p} \stackrel{\text { def }}{=}\left[1, a_{1}, \ldots, a_{p}\right]^{T}$ satisfy the Yule-Walker equations

$$
\hat{\mathbf{R}}_{c} \mathbf{a}_{p}=\epsilon_{p} \mathbf{u}_{1}
$$

where, $\hat{\mathbf{R}}_{c}$ is the (estimated) autocorrelation matrix of $c(t)$, $\epsilon_{p}$ is the modeling error, $\mathbf{u}_{1}=[1,0, \ldots, 0]^{T}$ is a unit vector of length $p+1$, and $p$ is the model order. Thus, the prediction recursion is given by

$$
\hat{c}(k)=-\sum_{l=1}^{p} a_{l} c(k-l)
$$

In practice, forward and backward linear prediction is applied for stability [6].

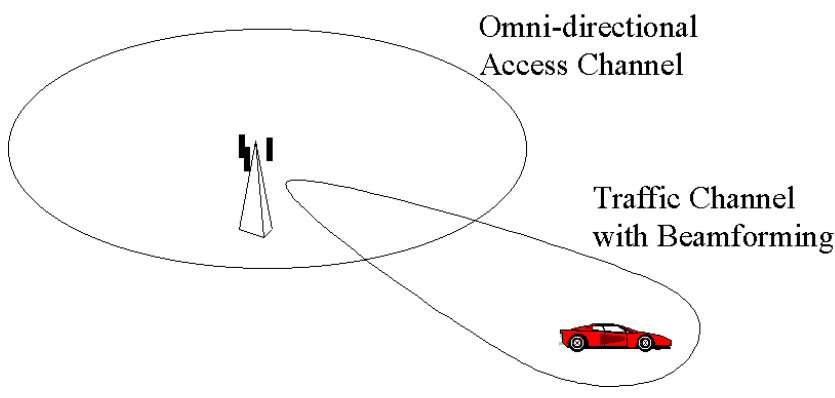

Figure 2. Access channel and traffic channel

Exploiting the BS antenna array, optimal beamforming weight vector $\mathbf{w}_{j}$ provides adequate signal quality for the desired mobile user while minimizing the interference from other user signals [7]. However, if the spatial signature $\mathbf{b}_{j}(t)$ changes rapidly, the downlink beamforming could actually result in received signal degradation. Consequently, the dedicated $\mathrm{TCH}$ for the mobile is sensitive to fast-fading, and it leads to large prediction error for uplink. The $\mathrm{ACH}$, on the other hand, is a broadcast channel, e.g. the universal sync channel, with $\mathbf{w}_{0}=\mathbf{1}$. Therefore, the ACH provides relative robustness to channel perturbation and reduces sensitivity to fast-fading (Fig. 2). From (11), the TCH and the 
$\mathrm{ACH}$ can be expressed as

$$
c_{T}(t)=\mathbf{w}_{j}^{T}[\mathbf{A}+\mathbf{D}] \Gamma, \quad c_{A}(t)=\mathbf{w}_{0}^{T}[\mathbf{A}+\mathbf{D}] \Gamma
$$

As discussed before, sharp peaks are the predominant feature of the power spectrums of $c_{T}(t)$ and $c_{A}(t)$. The corresponding peaks in the $\mathrm{TCH}$ and the $\mathrm{ACH}$ spectrums have the same locations, since $\mathbf{w}$ is a constant complex vector. This yields the same corresponding poles in both AR models. As a result, (normalized) prediction coefficients of the $\mathrm{TCH}$ and the ACH using AR models of the same order are the same. Along with (12), stacking TCH and ACH samples provides an overdetermined linear equation

$$
\left[\begin{array}{c}
\tilde{\mathbf{R}}_{T} \\
\tilde{\mathbf{R}}_{A}
\end{array}\right] \mathbf{a}_{p}=\mathbf{0}
$$

where, $\tilde{\mathbf{R}}_{T}$ and $\tilde{\mathbf{R}}_{A}$ are $p \times(p+1)$ autocorrelation matrices of TCH and ACH samples, respectively.

$\tilde{\mathbf{R}}=\left[\begin{array}{ccccc}r_{c}(1) & r_{c}(0) & r_{c}^{*}(1) & \cdots & r_{c}^{*}(p-1) \\ r_{c}(2) & r_{c}(1) & r_{c}(0) & \cdots & r_{c}^{*}(p-2) \\ \vdots & \vdots & \vdots & & \vdots \\ r_{c}(p) & r_{c}(p-1) & r_{c}(p-2) & \cdots & r_{c}(0)\end{array}\right]$

for both $\mathrm{TCH}$ and $\mathrm{ACH}$.

Due to channel randomness, $\left[\begin{array}{c}\tilde{\mathbf{R}}_{T} \\ \tilde{\mathbf{R}}_{A}\end{array}\right]$ usually has full column rank. Therefore, (15) has a unique non-trivial least squares solution with $\mathbf{a}_{p}(1)=1$.

\subsection{Dynamic Uplink Power Control}

Once the downlink symbols are detected, the mobile can extract its own $\mathrm{TCH} c_{T}(t)=\mathbf{w}_{j}^{T} \mathbf{b}_{j}(t)$ and $\mathrm{ACH} c_{A}(t)=$ $\mathbf{w}_{0}^{T} \mathbf{b}_{j}(t)$ from the received signal. Channel samples are passed through a low-pass-filter (LPF) to suppress noisy variation, and are then used to predict the TCH of the next uplink frame. The mobile transmitted power is adjusted according to the predicted uplink channel by the "waterfilling" scheme. The adjustment is dynamic in the sense that the transmitted power level changes as the channel changes during uplink. This can be expressed as

$$
P_{T X}(t)=P_{B T h}+P L-F(t)
$$

where, $P_{T X}(t)$ is the mobile transmitted power level in $\mathrm{dB}$, $P_{B T h}$ is the known BS received power threshold for this particular mobile user, $P L$ is the large-scale path loss which does not change during the short period of time under consideration, $F(t)$ is the uplink traffic channel fading function. In the fast-fading environment, $F(t)$ changes rapidly proportional to the envelope of the predicted uplink TCH. The scale of $F(t)$ is chosen in such a way that the average transmitted power of an uplink frame is fixed.
Note that the channel sampling rate utilized for dynamic uplink power adjustment is much lower than the data symbol rate. Mobile user adjusts transmitted power a few times during uplink in an effort to combat deep fades within a frame.

\section{Simulation}

A wireless CDMA system of a base station communicating with two mobile users in the propagation environment as in Fig. 1 is simulated. The BS has an 8-element uniform circular antenna array, which has half wavelength radius. We consider a typical vehicular scenario, in which the BS is located outdoors with a high antenna (thus no scatterers local to the BS), and the mobiles move at a speed of $80 \mathrm{~km} / \mathrm{h}$.

The received signals experience both local scattering and large-angle reflection. There are L dominant multipath bundles from each mobile arriving at the base station, with pass losses, delays and DOA's uniformly distributed on $[0 \mathrm{~dB}, 20$ $\mathrm{dB}],[0, T]$ and $[0,2 \pi]$, respectively. $\mathrm{T}$ is the chip duration. Chip rate is $2.048 \mathrm{MHz}$. Integer $\mathrm{L}$ is uniformly distributed on $[1,6]$. Each multipath bundle consists of $N=100$ local scattering paths. There is $10 \mathrm{~dB}$ AWGN at the receiver end. Communication is in TDD mode with $2.5 \mathrm{~ms}$ downlink and $2.5 \mathrm{~ms}$ uplink. Carrier frequency is $2 \mathrm{GHz}$. CDMA spreading factor is 8 . The prediction order is $p=12$. Assume perfect symbol detection at the BS.

Fig. 3 illustrates the TCH of each mobile user during the time period of a consecutive downlink and uplink. The downlink TCH is passed through a LPF with cut-off frequency $200 \mathrm{~Hz}$. The LPF output is represented by the dash line in the downlink. The dash line in the uplink period is the predicted TCH. Fig. 4 shows the DQPSK constellations of BS received signals from each user, as the frame-constant uplink transmitted power compares with the dynamic uplink power. The performance comparison of the frame-constant uplink power control and dynamic uplink power control is made in Fig. 5, in which it shows the average bit-error-rate (BER) of the received signals versus SNR at the BS. Fig. 6 shows the average BER as the mobile moves at different speed. Here, $\mathrm{SNR}=10 \mathrm{~dB}$. The performance of power control based on $\mathrm{ACH} / \mathrm{TCH}$ prediction is also compared with that of the prediction only with TCH samples. Clearly, the proposed method has the best performance over fast fading channels.

\section{Conclusion}

We have introduced an effective uplink power control scheme for wireless TDD systems under fast fading. The control intelligence is located at the mobile user end. The control is open-loop, as it is based on the prediction of the 
uplink channel. Prediction accuracy is improved by exploiting the antenna array technique. This mobile power control scheme aims at compensating channel fading fluctuation within a short period of time, usually several frame durations. And it can be combined with distributed iterative power control algorithms for optimal system performance.

\section{References}

[1] D. Astély and B. Ottersten. The effects of local scattering on direction of arrival estimation with MUSIC. IEEE Transactions on Signal Processing, 47(12):3220-3234, Dec. 1999.

[2] M. H. Hayes. Statistical Digital Signal Processing and Modeling. John Wiley and Sons, 1996.

[3] T. S. Rappaport. Wireless Communications. Prentice Hall, NJ, 1996.

[4] F. Rashid-Farrokhi, L. Tassiulas, and K. J. R. Liu. Joint optimal power control and beamforming in wireless networks using antenna arrays. IEEE Transactions on Communications, 46(10):1313-1324, Oct. 1998.

[5] B. Sklar. Rayleigh fading channels in mobile digital communication system part II: Mitigation. IEEE Communication Magzine, pages 103-109, July 1997.

[6] C. W. Therrien. Discrete Random Signals and Statistical Signal Processing. Prentice Hall, NJ, 1992.

[7] M. Torlak, G. Xu, B. L. Evans, and H. Liu. Fast estimation of weight vectors to optimize multi-transmitter broadcast channel capacity. IEEE Transactions on Signal Processing, 46(1):243-246, Jan. 1998.

[8] R. D. Yates. A framework for uplink power control in cellular radio systems. IEEE Journal on Selected Areas in Communications, 13(7):1341-1347, Sept. 1995.
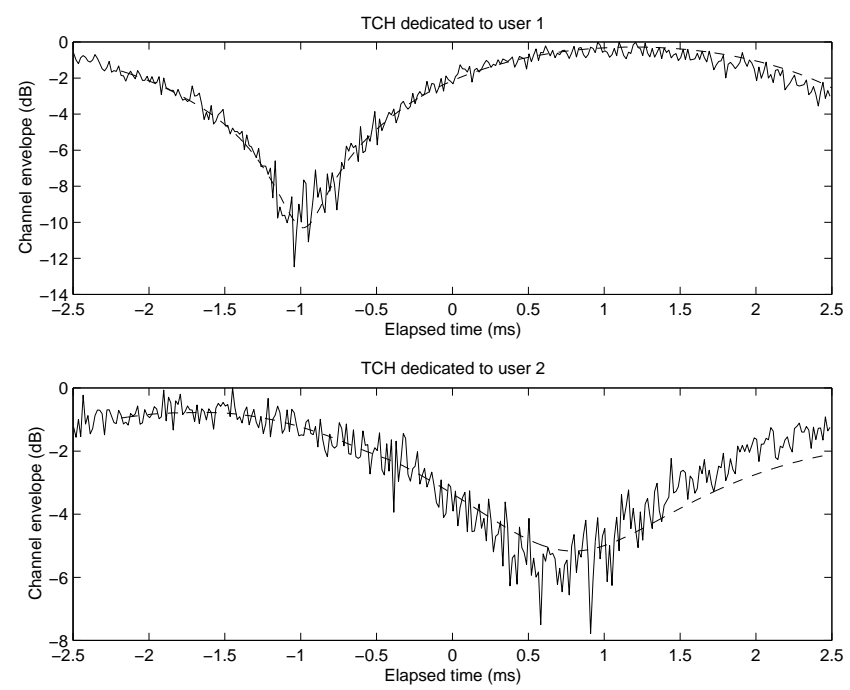

Figure 3. Downlink channel and (predicted) uplink channel.
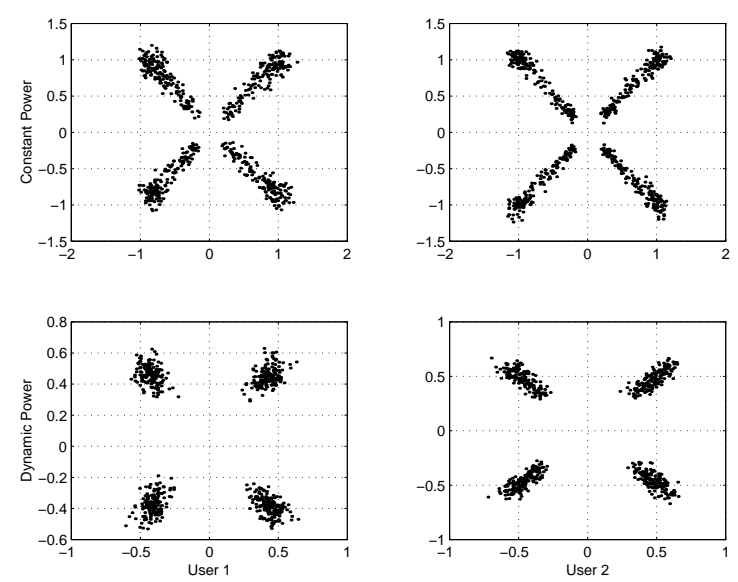

Figure 4. BS received signal constellation.

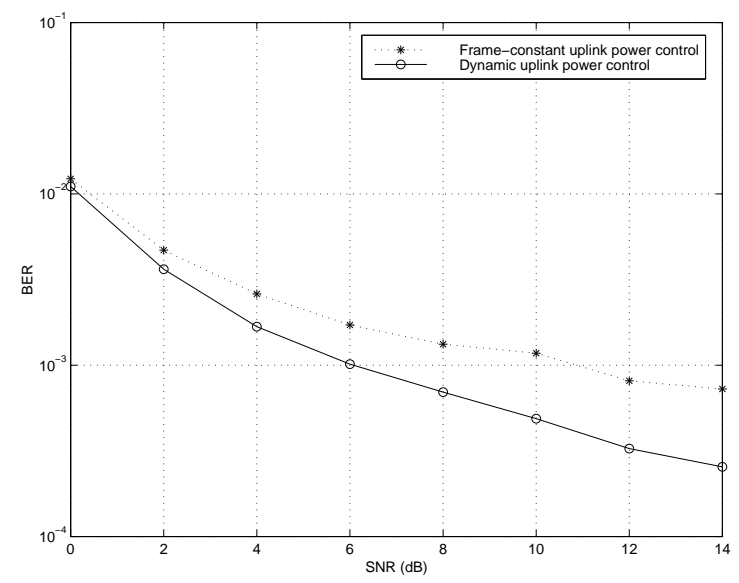

Figure 5. Performance of dynamic uplink power control.

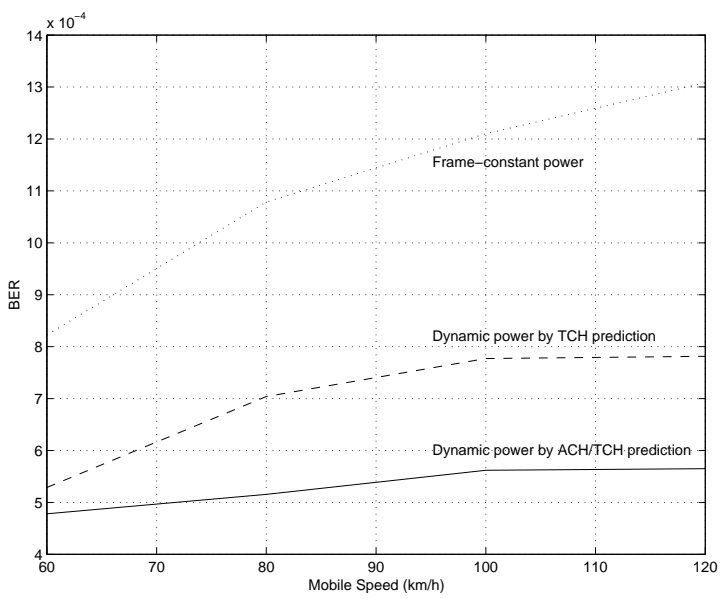

Figure 6. Performance of dynamic power control based on $\mathrm{TCH}$ or $\mathrm{ACH} / \mathrm{TCH}$ prediction. 